\title{
O IMPACTO DA MOBILIDADE DE RENDA SOBRE O BEM-ESTAR ECONÔMICO NO BRASIL *
}

\author{
Erik Alencar de Figueiredo ${ }^{\dagger}$
}

\begin{abstract}
Resumo
Em um estudo recente, Figueiredo e Ziegelmann (2007) apontam para os ganhos de bem-estar oriundos do aumento da mobilidade de renda no Brasil. Entretanto, seus resultados negligenciam um efeito deletério deste fenômeno, qual seja: o aumento da incerteza frente ao consumo futuro. Sob esta perspectiva, este estudo questiona a desejabilidade da mobilidade de renda no Brasil. Consideraram-se índices capazes de separar os seus efeitos negativo e positivo. Em suma, a partir de um cálculo para a aversão ao risco brasileiro, os resultados mostraram que a mobilidade só promoverá um aumento de bem-estar social, caso o parâmetro de aversão à desigualdade seja superior a 2.95 .
\end{abstract}

Palavras-chave: Mobilidade de renda, Desejabilidade, Bem-estar econômico.

\begin{abstract}
The study of Figueiredo e Ziegelmann (2007) showed that in Brazil there was a gain in welfare related to increasing of income mobility. However, this results did not consider one of the side effects related to this phenomenon: the increase of uncertainty associated to the behavior of consumption. According to this approach, this study questions how desirable is the income mobility in Brazil. It was used an index that separates the negative and positive effects related to mobility. The results showed, considering a coefficient of risk aversion, that an increase of income mobility will have positive effects only if the inequality parameter is superior to 2.95 .
\end{abstract}

Keywords: Income mobility, Desirability, Economic welfare.

JEL classification: I30, I39.

\footnotetext{
* Gostaria de agradecer o suporte financeiro do CNPq-Brasil. Estou em débito com o Editor Walter Belluzzo Jr e um parecerista anônimo. Erros e omissões são de minha inteira responsabilidade.

† Professor do Departamento de Economia da Universidade Federal da Paraíba.
} 


\section{Introdução}

A mobilidade de renda é socialmente desejável? As respostas para este questionamento dependerão da abordagem teórica considerada, podendo ser afirmativas ou incertas. Caso seja considerada que ela reduz a desigualdade nos múltiplos períodos, então a resposta será afirmativa, pois, de acordo com as estruturas teóricas tradicionais, tal como a sugerida por Atkinson (1981), a redução da desigualdade promove uma elavação do bem-estar. ${ }^{1}$ Porém, um aspecto relevante deve ser destacado: a mobilidade constitui uma importante fonte de incerteza. Sendo assim, seu impacto sobre o bem-estar torna-se ambíguo.

Sob a primeira linha de raciocínio, Gottschalk e Spolaore (2002) apontaram importantes ganhos de bem-estar associados ao aumento da mobilidade nas economias americana e alemã. E ainda, que estes ganhos foram mais expressivos nos EUA, devido a sua distribuição de renda mais desigual. Neste sentido, uma análise da realidade brasileira torna-se salutar, dado que este país encontra-se no topo do ranking das más distibuições de renda. ${ }^{2}$ Os parâmetros relacionados ao Brasil foram fornecidos por Figueiredo e Ziegelmann (2007) e seguiram a lógica dos resultados obtidos por Gottschalk e Spolaore (2002), ou seja, o país apresentou um alto ganho de bem-estar devido à mobilidade. Logo, surge uma conclusão direta: nações com grandes disparidades de renda tendem a beneficiar-se mais com o incremento da mobilidade.

Entretanto, com relação aos estudos mencionados, um ponto deve ser destacado: a estrutura teórica utilizada não admite perdas a priori relacionadas ao aumento da incerteza na economia. Assim, surge uma segunda linha de raciocínio, pela qual a desejabilidade da mobilidade é colocada em xeque.

A idéia é simples, para captar o impacto da mobilidade de renda sobre o bem-estar, dois efeitos devem ser considerados: um negativo, ex-ante, relacionado ao impacto da inclusão da incerteza na economia e; outro positivo, ex-post, associado à redução da desigualdade nos múltiplos períodos. Desta forma, o incremento de bem-estar só ocorreria se o segundo efeito dominasse o primeiro.

Guiado por este racioncínio, Abatemarco (2004) propõe uma abordagem teórica capaz de considerar, separadamente, estes efeitos. Para tanto, postulase a existência de um Observador Imparcial, nos moldes de Harsanyi (1953, 1955) e uma estrutura de otimização baseada no ciclo da vida. O modelo originado desta mistura permite que a aversão à incerteza seja determinada a partir de julgamentos éticos individuais. Com isso, torna-se possível captar as heterogeneidades, ou melhor, os padrões subjetivos de cada sociedade em relação à desejabilidade ou não da mobilidade.

Um exemplo claro destes padrões pode ser encontrado nos resultados de Alesina et al. (2001), pelos quais se observam impactos diferenciados da desigualdade sobre a felicidade dos indivíduos, quando se comparam as realidades européia e norte-americana.

Isto posto, o objetivo deste estudo é mensurar o efeito da mobilidade de renda sobre o nível de bem-estar econômico brasileiro. Para tanto, considerarse-á que o Observador Imparcial simpatiza com os interesses individuais $e x-$

\footnotetext{
${ }^{1}$ Este argumento desconsidera a questão da igualdade de oportunidades. Para detalhes sobre esta hipótese e sua relação com a mobilidade econômica, ver Van de Gaer et al. (2001).

2 Ver United Nations Development Program (2006).
} 
ante, sendo avesso à perda de bem-estar resultante da incerteza. Com isso, será possível isolar os efeitos positivos e negativos do movimento da renda.

Por fim, o artigo está organizado como segue. A segunda seção discute alguns conceitos básicos que servirão de referência para a discussão do modelo teórico apresentado na seção 3. A quarta seção é destinada à apresentação e discussão dos resultados. As considerações finais serão realizadas na quinta seção.

\section{Mobilidade de Renda e Bem-estar Econômico}

O estudo de Fields e Ok (2001) discute as múltiplas faces do conceito de mobilidade, demonstrando a inexistência de um discurso consensual na literatura. Porém, pode-se dizer, em poucas palavras, que a mobilidade de renda é a evolução da desigualdade ao longo do tempo. Neste sentido, é importante distinguir as duas dimensões deste fenômeno: i) a intergeracional, isto é, o papel da renda dos pais sobre a determinação da renda dos filhos; e, ii) a intrageracional referente ao quanto a renda do indivíduo no tempo $t$ pode interferir na sua renda em $t+1$.

A mobilidade intergeracional é normalmente explorada em estudos relacionados à igualdade de oportunidades. ${ }^{3}$ Neste campo de pesquisa, as teorias de justiça costumam associá-la diretamente aos ganhos de bem-estar (monotonicidade). Sendo assim, assume-se a mobilidade perfeita como ponto ótimo, dado que, sob este pressuposto, a origem do indivíduo não interferirá na sua renda futura. ${ }^{4}$

Do outro lado, o movimento intrageracional não representa uma boa medida para a igualdade de oportunidades, ${ }^{5}$ porém, a monotonicidade continua presente na maioria dos modelos teóricos. É fácil entender o porquê. Vejamos um exemplo: em seu livro Capitalism and freedom, Friedman (1962) afirma que:

(...) a given extent of income inequality in a rigde system in which each family stays in the same position in each period may be more a cause for concern than the same degree of inequality due to great mobility.

Logo, conclui-se que a persistência da desigualdade é mais grave do que seu nível. Neste contexto, a mobilidade de renda, reduzindo a desigualdade nos múltiplos períodos, sempre promoverá o aumento do bem-estar.

Entretanto, convém ressaltar que este tipo de análise negligencia um fator importante: o aumento na mobilidade gera incerteza na economia. A consideração deste fator pode mudar os resultados da monotonicidade, colocando em dúvida o efeito positivo da mobilidade sobre o bem-estar social. Tal abordagem pode ser encontrada, por exemplo, em Jarvis e Jenkins (1998), em que são listados os aspectos positivos e negativos da mobilidade.

De fato, sua desejabilidade dependerá de características subjetivas da sociedade, ou seja, de como cada uma avalia a desigualdade e o risco. Um exemplo claro de heterogeneidade pode ser encontrado em Alesina et al. (2001).

\footnotetext{
${ }^{3}$ Ver O’Neill et al. (2002).

4 Ver Prais (1955).

5 Ver de Gaer et al. (2001).
} 
Suas conclusões destacam que a desigualdade de renda possui um efeito negativo sobre a felicidade dos europeus mas não sobre a felicidade da população norte-americana, pois os americanos associam pobreza à ineficiência, enquanto que os europeus a consideram "falta de sorte".

Contudo, os resultados encontrados em Alesina et al. (2001) sugerem algo mais. Um olhar mais atento detecta que o grau de aversão à incerteza, captado pelos autores, surge na esfera do indivíduo a partir de julgamentos éticos. Em outras palavras, este tipo de aversão é formulado pela esfera privada e não por padrões sociais.

Neste ponto, há uma separação fundamental entre a proposta de Abatemarco (2004) e as abordagens que associam mobilidade intrageracional de renda e bem-estar econômico. ${ }^{6}$ Grosso modo, grande parte dos modelos baseiase em um Social Decision Maker (SDM). Sendo assim, a aversão à desigualdade e/ou incerteza que emergem destas estruturas não são resultantes de padrões éticos individuais.

Um exemplo pode ser encontrado no modelo desenvolvido por Atkinson e Bourguignon (1982), no qual não há incerteza quanto ao nível de renda futuro. Este parâmetro foi introduzido, posteriormente, por Gottschalk e Spolaore (2002). Entretanto, mesmo com esta modificação, ainda não foi possível isolar os efeitos deletérios da incerteza sobre o bem-estar, dado que uma de suas proposições postula que a aversão à desigualdade será sempre maior ou igual ao risco referente ao consumo futuro. ${ }^{7}$

Buscando contornar este problema, sugere-se a substituição da estrura SDM pelo Teorema do Observador Imparcial de Harsanyi $(1953,1955)$, mesclando-o com um modelo de ciclo da vida. Com isso, o parâmetro de aversão ao risco passa a ser determinado na esfera privada, a partir de padrões éticos dos agentes. Logo, as heterogeneidades sociais podem, a partir disso, ser captadas. Em suma, o modelo resultante permite mensurar: a perda de bemestar ex-ante devido ao aumento da incerteza na economia e o ganho ex-post oriundo da redução futura da desigualdade. Para um melhor entendimento desta estrutura teórica, convém apresentar algumas definições básicas na próxima subseção.

\subsection{Conceitos Básicos}

Nesta subseção, serão apresentados alguns conceitos econômicos citados anteriormente. O objetivo principal é discutir as implicações da inclusão do Teorema do Observador Imparcial.

A análise das utilidades individuais serão realizadas a partir de uma função de bem-estar social $W=F\left[U_{i}(X)\right]$, onde $F$ é definido como um agregador que, de acordo com Arrow (1994), envolve as "regras do jogo". Por exemplo, suponha que existam duas alternativas, $x$ e $y$, indicando dois vetores de renda, $x \neq y$. Desta forma, cada indivíduo $i$ escolherá $x$ se $U_{i}\left(x_{1}, \ldots, x_{n}\right) \geq U_{i}\left(y_{1}, \ldots, y_{n}\right)$

\footnotetext{
${ }^{6}$ De agora em diante a mobilidade intrageracional será tratada apenas por mobilidade.

7 O modelo de Gottschalk e Spolaore (2002) concentra-se na separação das duas fontes da mobilidade: a "reversibilidade" e a "independência da origem". A reversibilidade está associada ao grau em que as posições econômicas são revertidas ao longo do tempo, ou seja, a capacidade dos pobres tornarem-se ricos e os ricos tornarem-se pobres. Já a independência da origem diz respeito ao quanto a posição econômica atual depende da posição econômica no passado. Gottschalk e Spolaore (2002) demostram que o modelo de Atkinson e Bourguignon (1982) é compatível apenas com o primeiro conceito. Logo, ele não admite à existência de incerteza.
} 
e $F$ consistirá na soma não ponderada destas utilidades, gerando a seguinte função de bem-estar: $W=\sum_{i} U_{i}\left(x_{1}, \ldots, x_{n}\right)$.

Porém, a agregação de preferências pode não se dar de forma simples. Ou seja, ela pode envolver tensões comuns na literatura do bem-estar econômico, tais como o conflito entre os processos de escolhas pessoal e social. Neste caso, algum tipo de estrutura deve ser postulada.

No caso de um modelo com SDM, a função $W=U\left(x_{1}, \ldots, x_{n}\right)$ é diretamente imposta. Note que não há uma ponderação dos julgamentos individuais e sim a aceitação da utilidade $U$. Este tipo de utilidade é definida (de forma parcial ou completa) a partir de uma estrutura axiomática. ${ }^{8}$

Por fim, considere que a determinação de $F$ obedece o Teorema do Observador Imparcial de Harsanyi $(1953,1955)$. Em outras palavras, postula-se a existencia de um Observador Imparcial que tomará suas decisões tendo como base o conjunto de loterias da economia. Ao formar suas preferências, o Observador de Harsanyi assumirá a identidade de cada indivíduo $i$ da economia, considerando, portanto, suas relações de preferências (Principle of Acceptance). E mais, dada a racionalidade dos agentes, cada indivíduo $i$ tomará, ele mesmo, o papel do observador no momento da decisão. Desta forma, dentro da lógica de teoria bayesiana, cada agente escolherá o sistema social que maximize sua utilidade esperada, que nada mais é do que a média das utilidades de todos os agentes da economia:

$$
W=F\left[U_{i}(X)\right]=U(X)=\frac{1}{N} \sum_{j} u_{j}\left(x_{j}\right) .
$$

Neste contexto, o Principle of Acceptance toma lugar de destaque por associar a concavidade da função de bem-estar social a julgamentos éticos e não a situações de aversão social ao risco. Isto posto, considere que o Observador Imparcial simpatiza com os interesses individuais, dando atenção especial às perdas de bem-estar oriundas da incerteza.

Tendo estes conceitos em mente, considere uma economia onde uma população composta por indivíduos homogêneos, $N \equiv\{1, \ldots, n\}$, vivem dois períodos. Defina $x_{t}, x_{t+1} \in \mathfrak{R e}_{+}^{n}$ como os vetores de renda nos períodos $t$ e $t+1$, repectivamente, e o equivalente certeza no tempo $t$ por:

$$
C^{T}=\sum_{i} \pi_{i, t} C_{i}
$$

onde $\pi_{i, t}$ denota a distribuição marginal com respeito ao tempo $t$. Sendo assim, a função de bem-estar do ciclo da vida será:

$$
W=\sum_{i} \pi_{i, t} \omega\left(C_{i}\right)
$$

na qual $\omega$ é uma transformação côncava crescente. ${ }^{9}$ Considerando a abordagem isoelástica de Atkinson (1970), então

$$
W=\sum_{i} \frac{\pi_{i, t}}{1-\varepsilon}\left(x_{i, t}+x_{i, t(d \lambda)}\right)^{1-\varepsilon}
$$

\footnotetext{
${ }_{8}$ Esta estrutura é utilizada, por exemplo, por Atkinson (1970). Neste sentido, a concavidade de função de bem-estar e, por conseguinte, a aversão social à desigualdade, resulta do axioma da aversão individual ao risco.

${ }^{9}$ Assume-se um fator de desconto igual a um, por simplicidade.
} 
sendo $\varepsilon$ o parâmetro de aversão social à desigualdade e $\lambda$ representa a aversão ao risco. Focando no tempo $t+1$, teremos

$$
W=\sum_{i} \frac{\pi_{i, t}}{1-\varepsilon}\left(\sum_{j} \pi_{j \mid i} x_{j}^{1-\lambda}\right)^{\frac{1-\varepsilon}{1-\lambda}}
$$

onde $\pi_{j \mid i}$ é a probabilidade conjunta. É importante destacar que (2), sob algumas condições, torna-se equivalente à abordagem ex-ante proposta por Hammond $(1981,1983) .{ }^{10}$

Em resumo, a consideração do Observador Imparcial e do ciclo da vida resumido pelas funções (1) e (2) permitirá introduzir julgamentos éticos individuais como fundamento para a aversão ao risco. Já a aversão à desigualdade será calculada via equivalente certeza do ciclo da vida. Na seção seguinte será discutida a relação entre mobilidade de renda e bem-estar social ex-ante e ex-post.

\section{Mobilidade de Renda e Bem-estar ex-ante e ex-post}

Os resultados relativos aos efeitos ex-ante e ex-post serão obtidos a partir de uma função de bem-estar abreviada por $V[\mu, I]$, onde $\mu$ e $I$ correspondem, respectivamente, à média da renda e à desigualdade. Considerar-se-á $V[\mu, I]=$ $(1 / 2) \mu(1-I)$, sendo que $I$ corresponderá ao índice de Atkinson (1970).

A mobilidade será desejável se $W>W^{*}$ e, consequentemente, $V>V^{*}$, onde $W^{*}$ e $V^{*}$ representam a sociedade com perfeita imobilidade (estática) e $W$ e $V$ denotam o nível de bem-estar obtido a partir da renda vigente na sociedade. Vejamos essas considerações com maiores detalhes.

\subsection{Análise ex-post}

A observação do efeito ex-post, como já ressaltado, relaciona-se à interferência da desigualdade sobre o bem-estar. Sendo assim, este impacto pode ser captado pela comparação das medidas de desigualdade ao longo do tempo. De uma forma mais específica, comparando os níveis de desigualdade com e sem mobilidade, isto é $I\left(x_{t, t+1}\right)-I\left(x_{t, t}\right)$, onde $x_{t, t+1}=x_{t}+x_{t+1}$ e $x_{t, t}=x_{t}+x_{t}$. Considerando a homogeneidade de grau zero, teremos $I\left(x_{t, t+1}\right)-I\left(x_{t, t}\right)=I\left(x_{t, t+1}\right)-I\left(x_{t}\right)$, que corresponde ao efeito da mobilidade de renda proposto por Shorrocks (1978).

Da mesma forma, o efeito social da mobilidade pode ser captado pela diferença entre $U\left(x_{t, t+1}\right)-U\left(x_{t, t}\right)$. Considerando a função de bem-estar abreviada $V[\mu, I]=(1 / 2) \mu(1-I)$, a mensuração da desejabilidade social ex-post será: ${ }^{11}$

$$
D^{P}=\mu_{t, t+1}\left(I_{t, t+1}-I_{t, t}\right),
$$

com $\mu_{t, t+1}$ correspondendo à média do vetor de renda $x_{t, t+1}$. De forma equivalente (negligenciando a constante $1 / 2$ )

$$
D^{P}=x_{t, t+1}^{d(\varepsilon)}-x_{t, t}^{d(\varepsilon)}=x_{t, t+1}^{d(\varepsilon)}-2 x_{t}^{d(\varepsilon)},
$$

\footnotetext{
10 Para detalhes, ver a seção 3 de Abatemarco (2004). Em suma, as abordagens do SDM e do Observador Imparcial diferem na forma de considerar $\varepsilon$ e $\lambda$ na função de bem-estar.

11 Os subescritos $(\cdot)_{t, t+1}$ ou $(\cdot)_{t, t+1 d(\lambda)}$ significam que as variáveis estão sendo avaliadas ao longo do ciclo da vida. Por exemplo: $\mu_{t, t+1 d(\lambda)}$ representa a média da renda oriunda de $x_{t}+x_{t+1} \mathrm{e}$ considerando a aversão à incerteza $\lambda$.
} 
onde $x_{t, t+1}^{d(\varepsilon)}$ e $x_{t, t}^{d(\varepsilon)}$ são, respectivamente, os níveis de renda que vigorariam caso as distribuições fossem igualitárias, calculados a partir de $\left(x_{t}+x_{t+1}\right)$ e $\left(x_{t}\right)$. Sendo assim, dada uma média constante e uma mudança na mobilidade, $x_{t, t}^{d}$ será o menor valor para o indicador de bem-estar nos dois períodos, fazendo com que $D^{P}$ seja não-negativo. Em outras palavras, um incremento na mobilidade gerará sempre um ganho de bem-estar ex-post.

\subsection{Análise ex-ante}

Considere uma função de bem-estar abreviada para (2.1). Assim, o efeito da mobilidade pode ser definido por

$$
V-V^{*}=\mu_{t, t+1 d(\lambda)}\left(1-I_{t, t+1 d(\lambda)}\right)-\mu_{t, t}\left(1-I_{t, t}\right) .
$$

Ou similarmente,

$$
D_{C}^{a}=x_{t, t+1 d(\lambda)}^{d(\varepsilon)}-x_{t, t}^{d(\varepsilon)}
$$

$\operatorname{com} x_{t, t+1 d(\lambda)}^{d(\varepsilon)}$ indicando a renda que vigoraria caso a distribuição fosse igualitária, levando em conta os parâmetros $\varepsilon$ e $\lambda$.

A expressão (3) pode ser decomposta em dois fatores. O primeiro é relacionado ao efeito da incerteza, ou seja, mudança na média dada uma desigualdade constante, ou seja:

$$
D_{C}^{u}=\left(\mu_{t, t+1 d(\lambda)}-\mu_{t, t}\right) \frac{x_{t, t+1 d(\lambda)}^{d(\varepsilon)}}{\mu_{t, t+1 d(\lambda)}} .
$$

Pressupõem-se $\mu_{t, t+1 d(\lambda)}<\mu_{t, t}$, portanto, a primeira parte da expressão é não-positiva. O segundo fator diz respeito aos benefícios ex-ante da redução na desigualdade, isto é, da mudança na desigualdade dada uma média constante (como já ressaltado anteriormente, ele é não-negativo), sendo definido por:

$$
D_{C}^{i}=\left(\frac{x_{t, t+1 d(\lambda)}^{d(\varepsilon)}}{\mu_{t, t+1 d(\lambda)}}-\frac{x_{t, t}^{d(\varepsilon)}}{\mu_{t, t}}\right) \mu_{t, t} .
$$

Dessa forma, o efeito da incerteza $D_{C}^{u} \leq 0$ e da desigualdade $D_{C}^{i} \geq 0$. Isto quer dizer que a mobilidade de renda causa um efeito negativo, devido à incerteza, e um outro efeito positivo relacionado à redução da desigualdade.

\subsection{3. Índices}

Nas seções anteriores, observou-se que a inclusão da hipótese de um Observador Imparcial em conjunto com a análise do ciclo da vida permite avaliar, isoladamente, os efeitos ex-ante e ex-post da mobilidade. Nesta subseção, serão apresentados alguns índices úteis para a análise empírica. Neste contexto, dois índices tomam lugar de destaque: i) o ex-post

$$
D_{R}^{P}=\frac{x_{t, t+1}^{d(\varepsilon)}-x_{t, t}^{d(\varepsilon)}}{\mu_{t, t+1}}
$$

equivalente à medida sugerida por Shorrocks (1978), portanto, $D_{R}^{P} \in[0,1] \mathrm{e}$; ii) o ex-ante 


$$
D_{R}^{a}=\frac{x_{t, t+1 d(\lambda)}^{d(\varepsilon)}-x_{t, t}^{d(\varepsilon)}}{\mu_{t, t+1}},
$$

onde $D_{R}^{a} \in[-\infty, 1]$. O índice ex-ante pode ser decomposto em duas medidas:

$$
D_{R}^{u}=\frac{x_{t, t+1 d(\lambda)}^{d(\varepsilon)}}{\mu_{t, t}}-\frac{x_{t, t+1 d(\lambda)}^{d(\varepsilon)}}{\mu_{t, t+1 d(\lambda)}}, D_{R}^{i}=\frac{x_{t, t+1 d(\lambda)}^{d(\varepsilon)}}{\mu_{t, t+1 d(\lambda)}}-\frac{x_{t, t}^{d(\varepsilon)}}{\mu_{t, t}} .
$$

A interpretação destas medidas é simples. O indicador ex-post considera a monotonicidade, ou seja, quanto maior a mobilidade, maiores os ganhos de bem-estar. Já o índice ex-ante é decomposto em duas dimensões, uma reage positivamente ao aumento do bem-estar, dada a redução da desigualdade nos mútiplos períodos. A outra tem direção oposta, ou seja, a mobilidade gera incerteza, que, por sua vez, reduz o bem-estar.

\section{Aplicação}

Esta seção será reservada para os principais resultados do estudo. Em primeiro lugar, apontam-se a natureza e a construção do banco de dados. Em seguida, os índices de mobilidade serão apresentados e discutidos.

\subsection{Resultados}

Todos os resultados do estudo foram obtidos a partir da variável "renda pessoal de todos os trabalhos", coletada em dois momentos distintos, 1995 e 2005. Logo, está se considerando dez anos de mobilidade. As informações de renda foram extraídas da Pesquisa Nacional por Amostras de Domicílios (PNAD), realizada pelo Instituto Brasileiro de Geografia e Estatística (IBGE). Os valores estão expressos em Reais de janeiro de 2005. Para tanto, utilizou-se o procedimento sugerido por Corseuil e Foguel (2002).

A utilização de uma variável de renda envolve um importante debate na literatura empírica, qual seja: considerar a renda ou o consumo como indicador de bem-estar? Quando se trabalha com uma medida de renda corrente, supõe-se a existência de imperfeições no mercado de crédito. Neste caso, os agentes econômicos se veem impossibilitados de tomar empréstimos. Em resumo, argumenta-se que a trajetória do consumo pode ser mais suave ao longo do ciclo da vida, enquanto a renda está sujeita a variações sazonais. ${ }^{12}$ Contudo, a utilização da renda é uma prática comum nos estudos de mobilidade internacionais, dada a limitação referente à disponibilidade de dados de consumo. ${ }^{13}$

A amostra considera os chefes de família do sexo masculino com idade entre 25 e 54 anos em 1995 e vivendo em domicílios urbanos. Além disso, excluíram-se as rendas negativas, iguais a zero e quase-zero. Este procedimento foi utilizado, pois o cálculo de algumas medidas de desigualdade, entre elas o índice de Atkinson, apresenta relativa sensibilidade a estas observações. O método de exclusão das rendas quase-zero baseou-se na estatística proposta por Cowell et al. (1996), isto é,

\footnotetext{
12 Para detalhes, ver o capítulo 2 de Duclos e Araar (2006).

13 Podendo-se citar, entre outros, Gottschalk e Spolaore (2002) e Atkinson e Bourguignon (1982).
} 
Tabela 1: Impacto da Mobilidade sobre o Bem-estar Econômico no Brasil

\begin{tabular}{crrr}
\hline \multicolumn{5}{c}{ Indices ex-ante } \\
\cline { 2 - 4 } Total $\left(D_{R}^{a}\right)$ & $\varepsilon=0.5$ & $\varepsilon=1.5$ & $\varepsilon=3.0$ \\
\hline$\lambda=0.5$ & 0,8106 & 0,5067 & 0,3209 \\
$\lambda=1.5$ & $-0,1974$ & $-0,0031$ & 0,1235 \\
$\lambda=3.0$ & $-0,4123$ & $-0,1428$ & 0,0466 \\
\hline Efeito Positivo $\left(D_{R}^{i}\right)$ & $\varepsilon=0.5$ & $\varepsilon=1.5$ & $\varepsilon=3.0$ \\
\hline$\lambda=0.5$ & 0,8618 & 0,5375 & 0,3356 \\
$\lambda=1.5$ & 0,6148 & 0,4851 & 0,3570 \\
$\lambda=3.0$ & 0,3970 & 0,3474 & 0,2801 \\
\hline Efeito Negativo $\left(D_{R}^{u}\right)$ & $\varepsilon=0.5$ & $\varepsilon=1.5$ & $\varepsilon=3.0$ \\
\hline$\lambda=0.5$ & $-0,0512$ & $-0,0308$ & $-0,0147$ \\
$\lambda=1.5$ & $-0,8122$ & $-0,4882$ & $-0,2335$ \\
$\lambda=3.0$ & $-0,8093$ & $-0,4902$ & $-0,2335$ \\
\hline \multicolumn{5}{c}{ Índices ex-post } \\
\cline { 2 - 4 } 0,0318 & 0,1192 & 0,1717 \\
\hline$D_{R}^{P}$ & &
\end{tabular}

Fonte: Dados da pesquisa.

$$
I F(x, y)=\frac{x^{\alpha}+\sum_{i=1}^{n} w_{i} \frac{y_{i}^{\alpha}}{n}\left(\alpha-1-\frac{\alpha x}{\mu(y)}\right)}{\left(\alpha^{2}-\alpha\right) \mu(y)^{\alpha}} .
$$

Onde IF representa a função de influência dos dados sobre a medida de desigualdade. A variável $y$ diz respeito ao vetor de renda, $w$ o peso da observação, $x$ a renda mais baixa observada na distribuição, $\alpha=1-\varepsilon, n$ o tamanho da amostra e $\mu(y)$ a média do vetor de renda $y$. Os valores de $x$ associados a uma IF superior a $10 \%$ foram excluídos. Em média, esta exclusão não ultrapassou o percentual de $0,0005 \%$ da amostra em cada $\varepsilon$ considerado.

Os resultados relativos à desejabilidade da mobilidade de renda no Brasil, no período de 1995 a 2005, encontram-se sumarizados na Tabela 1. Os índices foram divididos em ex-ante e ex-post. Os indicadores ex-ante são separados em efeitos positivos, relacionados aos ganhos de bem-estar via redução da desigualdade, e negativos oriundos da perda de bem-estar devido ao aumento da incerteza na economia. Nesta primeira bateria de simulações, foram considerados três valores para os parâmetros de aversão à desigualdade $(\varepsilon)$ e aversão ao risco $(\lambda)$, são eles: $0.5,1.5$ e 3.0 .

A observação do índice ex-post $\left(D_{R}^{P}\right)$ indica que a mobilidade aumenta o bem-estar social. E ainda, este aumento é mais expressivo quanto maior for o grau de aversão à desigualdade. Note que o indicador passa de 0.0318 , com um $\varepsilon=0.5$, para 0.1717 , com $\varepsilon=3.0$. Este resultado corrobora as evidências apontadas por Gottschalk e Spolaore (2002) ao investigarem as realidades norte-americana e alemã, e Figueiredo e Ziegelmann (2007), abordando o caso brasileiro. Caso fosse observado apenas esta dimensão, poderia-se afirmar que a mobilidade de renda promove um incremento no bem-estar econômico, beneficiando, em maior escala, as nações com grandes iniqüidades de renda. 
Tabela 2: Impacto ex-ante da Mobilidade sobre o Bem-estar Econômico

\begin{tabular}{lccc}
\hline \multicolumn{3}{c}{ Índices ex-ante } \\
\hline \multicolumn{3}{c}{ Estimativas com $\lambda=4.89$} \\
\hline \multirow{3}{*}{$\varepsilon=0.5$} & $\varepsilon=1.5$ & $\varepsilon=3.0$ \\
\cline { 2 - 4 } Total $\left(D_{R}^{a}\right)$ & $-0,4952$ & $-0,2048$ & 0,0063 \\
\hline Fonte: Dados da pesquisa.
\end{tabular}

Contudo, a separação dos índices em valores ex-ante e ex-post serve para lançar dúvidas sobre este argumento. Note que o indicador ex-ante total $\left(D_{R}^{a}\right)$ mostra-se negativo para valores de $\lambda \geq 1.5$ e $\varepsilon \leq 1.5$, sugerindo que quanto maior a aversão ao risco, menor a desejabilidade da mobilidade de renda. Todavia, esta desejabilidade passa a ser controlada, também, pelo grau de aversão à desigualdade. As estatísticas $D_{R}^{i}$ e $D_{R}^{u}$ mostram as magnitudes dos componentes de $D_{R}^{a}$.

Os resultados são bastante intuitivos, mas não apresentam uma resposta definitiva para a questão da desejabilidade no Brasil, dado que foram considerados valores ad hoc de $\varepsilon$ e $\lambda$. Sendo assim, devem-se calibrar as funções a partir de parâmetros estimados para a economia brasileira, principalmente no que se refere a aversão ao risco, pois o modelo teórico postula sua obtenção a partir de características subjetivas da sociedade.

Felizmente, o estudo de Issler e Piqueira (2000) apresenta as estimativas para o grau de aversão ao risco brasileiro. Embora seus resultados sejam inconclusivos por dependerem da forma da função utilidade considerada. Em resumo, para uma função CRRA ( constant relative risk aversion) e dados anuais, o $\lambda=4.89$. Para outras estruturas e frequências de dados este parâmetro situou-se entre $0.5 \leq \lambda \leq 1$, indicando uma neutralidade ao risco. Contudo, utilizando critérios técnicos de estimação, os autores argumentam em favor da alta averssão.

A Tabela 2 apresenta os resultados sintéticos considerando o parâmetro de aversão ao risco calculado por Issler e Piqueira (2000), $\lambda=4.89$. Entretanto, na falta de estimativas para $\varepsilon$, cosideraram-se valores ad hoc. Em suma, os resultados sugerem que a mobilidade de renda só será socialmente desejável se a aversão à desigualdade brasileira for superior a 1.5. Na verdade, com um $\lambda=4.89$, este threshold dá-se a partir de $\varepsilon=2.95$.

Estes resultados conduzem a uma importante reflexão. Em estudos relacionados, respectivamente, às mobilidades intergeracional e intrageracional, Ferreira e Veloso (2006) e Figueiredo e Ziegelmann (2008) constataram que o Brasil apresenta uma elevada rigidez em sua estrutura social. Neste contexto, o aumento da mobilidade de renda passa a representar uma "quebra" nesta estrutura, gerando, com isso, uma melhor distribuição dos rendimentos e, por conseguinte, uma situação caracterizada pelo maior nível de bem-estar econômico.

Contudo, a partir das evidências apontadas nesta seção, pode-se restringir esta conclusão, ou seja: o aumento da mobilidade de renda só propiciará uma elevação do bem-estar, ou melhor, só será socialmente desejável, se a aversão à desigualdade de renda for superior a 2.95. Resumindo, a mobilidade de renda introduz um importante fator de incerteza na economia e, dada a alta aversão 
ao risco futuro observado no país, ela só gerará um aumento de bem-estar se for observado um alto grau de aversão à desigualdade.

\section{Considerações Finais}

Ferreira e Veloso (2006) e Figueiredo e Ziegelmann (2008) apontam, em estudos recentes, para a elevada rigidez da estrutura social brasileira. Sob esta constatação, Figueiredo e Ziegelmann (2007) concluem que o Brasil tende a se beneficiar com o aumento da mobilidade, pois a "quebra" desta estrutura promoverá um aumento no bem-estar social.

Tendo estes fatos como suporte, esta pesquisa questionou: a mobilidade de renda no Brasil é socialmente desejável? A obtenção das respostas passou, necessariamente, pelo abandono das estruturas teóricas tradicionais. Ou seja, começou a se duvidar do caráter monotônico da mobilidade.

Em resumo, observou-se que a mobilidade, apesar de reduzir a desigualdade nos múltiplos períodos, gera uma importante fonte de incerteza na economia. Logo, a captação do seu o impacto sobre o bem-estar social deve considerar dois efeitos: um negativo, ex-ante, relacionado à inclusão da incerteza na economia e; outro positivo, ex-post, associado à redução da desigualdade nos múltiplos períodos.

As calibrações dos índices ex-ante e ex-post indicaram que quanto maior a aversão à desigualdade e menor a aversão ao risco, mais desejável será a mobilidade de renda. Ao considerar a estimativa para a aversão à desigualdade contida em Issler e Piqueira (2000), isto é, um $\lambda=4.89$, conclui-se que a mobilidade de renda só aumentará o nível de bem-estar social se o parâmetro de aversão à desigualdade for superior a $\varepsilon=2.95$.

Por fim, o estudo deixa algumas importantes lacunas. A primeira diz respeito ao não conhecimento do grau de aversão à desigualdade brasileiro. Sendo assim, o próximo passo será dado em busca destas estimativas. Outro ponto relevante é a aplicação deste instrumental aos dados norte-americanos e alemães criando, com isso, uma importate fonte de captação das heterogeneidades sociais e servindo como base de comparação para as evidências de Alesina et al. (2001).

\section{Referências Bibliográficas}

A. Abatemarco. Is income mobility socially desirable? Technical report, University of Napoli, 2004.

A. Alesina, R. Di Tella, e R. MacCulloch. Inequality and happiness: are europeans and americans different? Technical report, NBER, 2001.

A. Atkinson. On the measurement of inequality. Journal of Economic Theory, 2:244-263, 1970.

A. Atkinson. The measurement of mobility. In A. B. Atkinson, editor, Essays in honor of Jan Pen. Brighton: Wheatsheaf, 1981.

A. Atkinson e F. Bourguignon. The comparison of multidimensional distribuition of economic status. Review of Economic Studies, 49:183-201, 1982. 
C. Corseuil e M. Foguel. Uma sugestão de deflatores para rendas obtidas a partir de algumas pesquisas domiciliares do ibge. Mercado de Trabalho Conjuntura e Análise, 1(19), 2002.

F. Cowell, V.Feser, e M. Pia. Robustness properties of inequality measures. Econometrica, 64:77-101, 1996.

D. Van de Gaer, E. Schokkaert, e M. Martinez. Three meaning of intergeneration mobility. Economica, 68:519-537, 2001.

J-Yves Duclos e A. Araar. Poverty and equity: measurement. policy, and estimation with DAD. New York: Springer, 2006.

S. Ferreira e F. Veloso. Intergenerational mobility of wages in brazil. Brazilian Review of Econometrics, 26:181-211, 2006.

G. Fields e E. Ok. The measurement of income mobility: an introduction to the literature. In J. Silber, editor, Handbook on income inequality measurement. Boston: Kluwer Academic Press, 2001.

E. Figueiredo e F. Ziegelmann. Mobilidade de renda e bem-estar econômico no brasil. Master's thesis, UFRGS, 2007.

E. Figueiredo e F. Ziegelmann. The dynamics of the brazilian income. Master's thesis, UFRGS, 2008.

M. Friedman. Capitalism and freedom. Princeton University Press, 1962.

P. Gottschalk e E. Spolaore. On the evalution of economic mobility. Review of Economic Studies, 69:191-208, 2002.

P. Hammond. Ex-ante and ex-post welfare optimality under uncertainty. Economica, 48:235-250, 1981.

P. Hammond. Ex-post optimality as a dynamically consistent objective for collective choice under uncertainty. In P. Pattanaik e M. Salles, editores, Social choice and welfare. North-Holland, 1983.

J. Harsanyi. Cardinal utility in welfare economics and in the theory of risktaking. Journal of Political Economy, 61:434-435, 1953.

J. Harsanyi. Cardinal welfare, individualistic ethics and interpersonal comparisons of utility. Journal of Political Economy, 63:309-321, 1955.

J. Issler e N. Piqueira. Estimating relative risk aversio, the discount rate, and the intertemporal elasticity of substitution in consumption for brazil using three types of utility function. Brazilian Review of Econometrics, 20:200-238, 2000.

D. O’Neill, O. Sweetman, e D. Van de Gaer. Equality of oportunity and kernel density estimation: an aplication to intergenerational mobility. Technical report, University of Maynooth, 2002.

S. Prais. Measuring social mobility. Journal of Royal Statistical Society, A: 56-66, 1955.

A. Shorrocks. Income inequality and income mobility. Journal of Economic Theory, 19:376-393, 1978. 